\title{
Otitis Media: Genetic Factors and Sex Differences
}

\author{
Ellen Kvestad', Kari J. Kværner², Espen Røysamb', Kristian Tambs', Jennifer R. Harris', and Per Magnus' \\ ' Division of Epidemiology, The Norwegian Institute of Public Health, Oslo, Norway \\ ${ }^{2}$ Center for Health Administration, the National Hospital, University of Oslo, Norway
}

A Ithough genetic factors are recognised as major contributors to otitis media, the presence of sex differences in heritability needs clarification. The aim of this study was to estimate the relative contribution of genetic and environmental effects in otitis media liability with particular focus on sex differences. Data from a cohort of Norwegian twins born between 1967 and 1979 with repeated measures on recurrent childhood otitis media were analysed. Altogether the sample included 4247 twin pairs. The tetrachoric correlations for monozygotic twins were .71 and .65 for males and females respectively. In dizygotic twins the correlations were .35 and .25 for males and females, respectively, and was .34 in opposite sexed pairs. The contribution of genetic and environmental effects was analyzed using structural equation modeling. The best fitting model showed that additive genetic effects explained $72 \%$ and $61 \%$ of the variance in males and females, respectively. The remaining variance was attributed to individual environmental effects. A model specifying equal heritability estimates for males and females yielded an almost equivalent fit. We found substantial genetic effects for liability to otitis media. There is no evidence that different sets of genes influence liability in males and females, but there may be sex differences in the relative importance of genetic effects.

Otitis media causes significant childhood morbidity and has an impact upon general public health (Hartman et al., 2001; Kvaerner et al., 2002; Niemela et al., 1999). Diverse lines of genetic research, ranging from quantitative to molecular studies, implicate the influence of genes in the pathogenesis of otitis media.

Twin studies report a large genetic predisposition to the disease (Casselbrant et al., 1999; Kvaerner et al., 1997; Rovers et al., 2002). Several studies suggest that multiple genetic factors may play a role in susceptibility or resistance to otitis media. For example, genetic loci may confer susceptibility to infections with certain bacteria and subsequently cause development of persistent and recurrent otitis media (Jaatinen et al., 1999; Lahti et al., 2002; Lofgren et al., 2002). Abnormalities in genes that influence anatomy of the skull or the immune defense system, such as ciliary movement and mucus production, would also increase the risk for ear infections. Current advances in molecular biology are accompanied by a growing understanding of the benefits of classical twin studies to understand otitis media (Daly et al., 2002). Twin studies can improve the efficiency of quantitative trait-loci detection as well as play an important role in unraveling developmental genetic mechanisms. For instance, such applications may be of value for identifying genes associated with cytokines and chemokines that have been shown to increase susceptibility to a number of infectious diseases (Joki-Erkkila et al., 2002).

Although three twin studies report genetic factors contribute substantially to otitis media liability (Casselbrant et al., 1999; Kvaerner et al., 1997; Rovers et al., 2002), results concerning sex specific effects are inconsistent (Kvaerner et al., 1997; Rovers et al., 2002). A Pittsburgh study (Casselbrant et al., 1999) used a clinical sample of 135 twin and 5 triplet pairs, measuring duration of middle ear effusion during the first 2 years of life. Heritability estimates were obtained using the De Fries and Fulker regression model. Heritability for time with middle ear effusion was $73 \%$, but the small sample size precluded estimation of sex differences. A British study (Rovers et al., 2002) collected prospective questionnaire data on 1373 samesex twin pairs with parental report data at age 2, 3 and 4 years. Nine different items regarding acute ear infection and upper airway blockage were analyzed separately using structural equation ACE models. Regarding acute ear infections, additive genetic effects explained $57 \%$ of the variance while common environmental effects explained $18 \%$. The remaining variance was explained by individual environment. This study of same-sex twins did not report significant sex differences. In the previous study of 2750 Norwegian twin pairs, structural equation modeling revealed a sex difference in the heritability estimates (Kvaerner et al.,

Received 15 September, 2003; accepted 29 January, 2004.

Address for correspondence: Ellen Kvestad, Division of Epidemiology, The Norwegian Institute of Public Health, Pb 4404 Nydalen, N-0403 Oslo, Norway. Email: ellen.kvestad@fhi.no 
1997). In males, additive genetic effects explained $45 \%$ of the variance and common environment explained $29 \%$ of the remaining variance. In females, additive and dominant genetic effects explained $74 \%$ of the variance. Collectively, the small body of literature on the heritability of otitis media suggests evidence for sex differences in the magnitude of genetic influences and more thorough investigation of the nature of these sex differences is needed.

The present study builds upon the previous Norwegian study (Kvaerner et al., 1997) and analyzes the relative contribution of genetic and environmental effects in otitis media liability on a larger sample of twins, with particular focus on sex differences in both genetic source and in the magnitude of the genetic estimates.

\section{Materials and Methods \\ Sample}

The Norwegian Institute of Public Health Twin Study is a longitudinal study with a cohort sequential design whereby new cohorts are planned to be recruited into the study at 5-6 year intervals. The current database includes information on twins born between 1967 and 1979, identified through the Medical Birth Registry of Norway. Two questionnaire studies have been conducted so far, in 1992 (questionnaire 1 [q1]) and 1998 (questionnaire 2 [q2]), with individual response rates of $73 \%$ and $63 \%$, and pairwise response rates of $64 \%$ and $52 \%$, respectively. Q1 was sent to all twins born between 1967 and 1974, who were at least 18 years old and for whom a current address in Norway was obtained. Q2 was sent to all twins who had received q1, plus to five new birth cohorts including twins born between 1975 and 1979. The combined q1 and q2 sample includes 9479 twins who responded to at least one of the questionnaires, with 4430 twins participating in both studies. Table 1 shows the number of pairs participating in $\mathrm{q} 1, \mathrm{q} 2$ and the combined sample by sex and zygosity. In $\mathrm{q} 1$ and $\mathrm{q} 2$ there were 724 and 1377 pairs respectively from whom only one twin responded. Pairs where one twin has responded to $\mathrm{q} 1$ and the co-twin

\section{Table 1}

Number of Pair Responders by Sex and Zygosity

\begin{tabular}{lccc}
\hline Group & $\begin{array}{c}01 \\
\text { Pairs }(M)\end{array}$ & $\begin{array}{c}02 \\
\text { Pairs }(M)\end{array}$ & $\begin{array}{c}\text { Combined Sample } \\
\text { Pairs }(M)\end{array}$ \\
\hline MZM & 416 & 526 & 677 \\
MZF & 528 & 777 & 904 \\
DZM & 387 & 397 & 592 \\
DZF & 443 & 655 & 789 \\
DZU & 796 & 979 & 1285 \\
total & 2570 & 3334 & 4247 \\
\hline
\end{tabular}

Note: 01, 1992 questionnaire; 02, 1998 questionnaire; MZM, monozygotic males; MZF, monozygotic females; DZM, dizygotic males; DZF, dizygotic females; DZUm, dizygotic unlike sex males; DZUf, dizygotic unlike sex females. has responded to $\mathrm{q} 2$ or vice-versa are treated as pair responders in the combined sample. The twin research program, including procedures and zygosity determination, is described in detail elsewhere (Harris et al., 1995; Harris et al., 2002).

\section{Measures}

Both questionnaires included the following items about ear infections: "Do you have or have you had recurrent infections in the ear? If yes, do you remember age of onset of the first infection?" The responders were asked to provide the specific age of onset of the first ear infection.

Data from twins who have answered both questionnaires can be utilized for an examination of reporting inconsistency; for example, otitis media may be reported in $\mathrm{q} 1$, but not confirmed in $\mathrm{q} 2$ or vice-versa. Test-retest tetrachoric correlation between the response in the two data sets was .82 . For this study, we assume that those who report ear infections at least once have had the disease. Accordingly, all individuals reporting recurrent ear infections with onset before age 7 years in at least one questionnaire were included as cases. Respondents with recurrent ear infections and missing data on age at onset were included in the analyses as cases. Estimated test-retest reliability and reporting consistency, elucidating the validity of the measure, is presented in a forthcoming publication (Kvestad et al., in preparation).

\section{Analysis}

Data from complete pairs were used to calculate the distribution of recurrent otitis media by zygosity and sex. To estimate the relative contribution of genes and environment in the liability of recurrent otitis media, we used the computer program Mx for structural equation modeling (Neale et al., 1999). This method is frequently used in twin data analysis and is described in detail elsewhere (Neale \& Cardon, 1992). Co-twin similarity was calculated using tetrachoric correlations for the five groups, defined by sex and zygosity, and were estimated using the computer program PRELIS2 (Jöreskog \& Sörbom, 2000). This computation is based on the assumption of an underlying normally distributed liability to recurrent otitis media. Manifest disease occurs when a certain threshold value is reached. Using these models variation in the liability to recurrent otitis media is decomposed into unobserved latent factors. Genetic factors comprise additive $(a)$ and dominant $(d)$ effects. Additive genetic effects refer to the sum of the average effects of the individual alleles at all loci. Dominant effects are sums of intra-locus interactions in which one allele has more influence on phenotypic expression than the other allele in individuals carrying two different alleles at one locus and refers to the extent to which genotypic values differ from the expected additive genetic values. Identical twins share all their genes whereas fraternal twins share, on average, half of their segregating genes. 
Table 2

Prevalence of Recurrent Ear Infections Prior to Age 7, Distribution of Concordant and Discordant Pairs and Tetrachoric Correlations in a Study of 4247 Twin Pairs, According to Zygosity and Sex

\begin{tabular}{lcccccc}
\hline Group & Pairs $(M)$ & Concordant pairs $(n)$ & Discordant pairs $(n)$ & Prevalence $(\%)$ & $95 \%$ Cl & Tetrachoric correlation \\
\hline MZM & 677 & 29 & 66 & 9.2 & $7.6-10.7$ & .713 \\
MZF & 904 & 61 & 137 & 14.3 & $12.7-15.9$ & .645 \\
DZM & 592 & 12 & 82 & 9.0 & $7.3-10.6$ & .353 \\
DZF & 789 & 21 & 150 & 12.2 & $10.6-13.8$ & .248 \\
DZU & 1285 & 32 & 210 & 10.7 & $9.5-11.9$ & .335 \\
Total & 4247 & 255 & 645 & 11.2 & $10.5-11.9$ & \\
\hline
\end{tabular}

Note: MZM, monozygotic males; MZF, monozygotic females; DZM, dizygotic males; DZF, dizygotic females; DZU, dizygotic unlike sex; Cl, confidence interval.

Thus, identical twins are perfectly correlated for effects due to genetic additivity and dominance, and fraternal twins are correlated .5 for genetic additivity and .25 for genetic dominance. Environmental factors are partitioned into common $(c)$ and specific (e) environment. Common environment refers to environmental factors shared by the twins and are therefore perfectly correlated in both zygosity groups. Specific environmental factors contain all environmental factors not shared by co-twins as well as measurement error. Specific environmental factors contribute to differences within a pair and are thus uncorrelated for all pairs.

Because $(c)$ and $(d)$ cannot be simultaneously estimated in the structural models, the model fitting procedures compared full ACE and ADE models with nested submodels. The model was also expanded to test 2 types of sex-specific effects. In a common sexlimitation model, the same genes contribute to the variation in the phenotype but the magnitude of genetic and environmental effects is allowed to vary across sexes. A general sex-limitation model allows for differences in both genetic source and magnitude of variance estimates for males and females.

The goodness-of-fit for the different models were evaluated according to Akaike's Information Criterion (AIC $=\chi^{2}-2 \mathrm{df}$ ) (Akaike, 1987), addressing both likelihood and simplicity of models and allowing non-nested models to be compared.

\section{Results}

Recurrent ear infections before age 7 years was reported by $11.2 \%$ (955 persons). There was a significant predominance of female cases $13.1 \%$ (95\% CI, 12.2, 14.1) compared to males $8.9 \%$ (95\% CI, 8.0, 9.8). Distribution by sex and zygosity is shown in Table 2.

\section{Concordance and Tetrachoric Correlations}

Table 2 shows the number of cases and the distribution of pairs discordant and concordant for recurrent ear infections by zygosity and sex. As illustrated in the table, tetrachoric correlations for all MZ pairs are greater than for $\mathrm{DZ}$ pairs. The DZ unlike-sex correlation is not significantly smaller than the samesex DZ correlation.

\section{Model Fitting}

Table 3 shows the results of the model fitting procedure. The results are grouped according to genetic and environmental constraints; common sex-limitation models (models 1-3) and no sex-limitation models (models 4-6). Because the DZ unlike-sex correlation is not significantly smaller than the same-sex DZ correlations, there was no evidence that different genes or common environments contribute to otitis media liability in males and females. Accordingly, general sex-limitation models were not tested in the model fitting procedure.

The models listed in Table 3 are presented starting with the least constrained model, subsequently adding

Table 3

Results of Structural Equation Analysis of Tetrachoric Correlations in Childhood Recurrent Otitis Media in Twins

\begin{tabular}{lccccccccccccc}
\hline $\mathrm{Nr}$ & Model & $a^{2}{ }_{m}$ & $a^{2}{ }_{f}$ & $d^{2}{ }_{m}$ & $d^{2}{ }_{f}$ & $c^{2}{ }_{m}$ & $c^{2}{ }_{f}$ & $e^{2}{ }_{m}$ & $e^{2}{ }_{f}$ & $\chi^{2}$ & $d f$ & $p$ & AIC \\
\hline 1 & ADE & 0.69 & 0.55 & 0.04 & 0.07 & & & 0.27 & 0.38 & 0.95 & 1 & .33 & -1.05 \\
2 & ACE & 0.72 & 0.60 & & & 0.00 & 0.00 & 0.28 & 0.40 & 0.78 & 1 & .38 & -1.22 \\
3 & AE & 0.72 & 0.61 & & & & & 0.28 & 0.39 & 1.04 & 3 & .79 & -4.96 \\
4 & ADE & 0.61 & 0.61 & 0.06 & 0.06 & & & 0.33 & 0.33 & 3.18 & 3 & .36 & -2.82 \\
5 & ACE & 0.66 & 0.66 & & & 0.00 & 0.00 & 0.34 & 0.34 & 3.26 & 3 & .35 & -2.74 \\
6 & $\mathrm{AE}$ & 0.66 & 0.66 & & & & & 0.34 & 0.34 & 3.26 & 4 & .52 & -4.74 \\
\hline
\end{tabular}

Note: AIC, Akaike's Information Criterion; df, degrees of freedom; $a$, additive genetic effects; $d$, dominant genetic effects; $c$, common environment; e, non-shared environment. Subscripts ${ }_{f}$ and ${ }_{m}$ refer to females and males respectively. Reported effects are variance estimates (e.g., the values in the $a^{2}{ }_{m}$ column represent percentage of variance explained by the male additive genetic effects). Models 1-3 are common sex-limitation models, models $4-6$ are no sex-limitation models. 
constraints and comparing more parsimonious models to the full model. In model 2, the common environment $(c)$ was estimated close to zero for both males and females. The non-significance of the cparameter is reflected in the Chi-square difference between models 2 and $3\left(\Delta \chi^{2}=.26\right)$. The saturated model including genetic dominance (ADE) did not yield a better fit, using the AIC criterion, than the more parsimonious common sex-limitation AE model (model 3). When models in the no sex-limitation category were tested, common environmental effects, $(c)$ was again estimated close to zero. Accordingly, the AE model yielded best fit on this category.

In our study the fit of the $\mathrm{AE}$ model with common sex-limitation (model 3) and with no sex-limitation (model 6) were almost equivalent according to AIC (AIC values -4.96 and -4.74 , respectively). Further, the magnitude of genetic and environmental effects was almost identical in the two models. Ninety-five percent confidence intervals for each estimate given in parentheses; $a_{m}^{2}=.72(.61-.84) a_{f}^{2}=.61(.51-.71)$ $e^{2}{ }_{m}=.28(.16-.40)$ and $e^{2}=.39(.28-.50)$ for model 3 and $a_{m}^{2}=a^{2} .66(.58-.73)$ and $e_{m}^{2}=e^{2} .34$ (.26-.42) for model 6, respectively. Model 3 did not yield a significantly better fit than the more parsimonious model 6 when $\chi^{2}$ differences were compared.

\section{Discussion}

Sex differences in the genetic and environmental variance structure for liability to otitis media were explored using classical twin analysis with a large data material. The present study confirms earlier reports of a large genetic predisposition for otitis media (Casselbrant et al., 1999; Kvaerner et al., 1997; Rovers et al., 2002). Our results provide evidence for sex differences in the magnitude of the genetic effect.

\section{Genetic and Environmental Effects}

Our finding of a large heritability component is in agreement with other studies (Casselbrant et al., 1999; Kvaerner et al., 1997; Rovers et al., 2002). Genetic effects explained approximately $70 \%$ of the otitis media liability; the remaining variability was identified as specific environment. In the former Norwegian study, genetic effects were estimated at $45 \%$ and $74 \%$ for males and females, respectively. Common environmental effects explained $29 \%$ of the remaining variance in males. A substantial genetic effect in males and females from the same genetic source was confirmed in both studies. While agreement was found in the estimate of the female genetic effect, a larger genetic male effect has replaced the common environmental factor identified in the former Norwegian study, comprising fewer twin pairs. When looking at the pairs not participating in the former Norwegian study separately, the tetrachoric correlations are all in the same range as in the former Norwegian study except from the group of dizygotic males. In this group the correlation is estimated to be less than half the monozygotic male correlation. This decreases the dizygotic male tetrachoric correlation in the combined sample and explains why the male common environmental effects are not detectable in the present study.

The heritability estimates of the present study are essentially in agreement with genetic estimates on acute ear infections in the study by Rovers et al. (Rovers et al., 2002), where the genetic liability was estimated at $57 \%$. In the same study there was a significant effect of common environment explaining $18 \%$ of the remaining variance. The study by Casselbrant et al. (1999) estimated the genetic contribution to long-lasting middle ear effusion to $72 \%$. The overall agreement in the magnitude of the genetic effect suggests that differences in study samples, dependent variables and statistical methods applied have limited influence on the heritability estimates.

In contrast to the rather consistent observation of a considerable heritability for ear infections, we did not find evidence for common environmental effects as reported in the British study of preschool children (Rovers et al., 2002). Although this discrepancy may be due to substantial population differences concerning risk factor exposure or genes or to random fluctuation, it is likely that differences in methodology may explain this discrepancy. While the retrospectively reported information may be subject to recall bias and a reduction in the magnitude of genetic effects due to measurement errors, as discussed in the former Norwegian twin study (Kvaerner et al., 1997), prospective information may artificially increase common environmental effects. Parental reporting on current illness in twins may introduce an artificially increased intra pair similarity in both monozygotic and dizygotic twins. There may both be a tendency for more similar reporting and a lower threshold for physician diagnosed otitis media in the co-twin if the first twin experiences recurrent ear infections. Subsequently, intra pair correlations will be increased and favor a model including common environment at the expense of genetic dominance. A similar nonblinded reporting bias is commented upon in the Pittsburgh study (Casselbrant et al., 1999).

\section{Sex Differences}

The question of sex differences in the heritability of otitis media has been raised in the literature, but thus far, studies have been unable to resolve this issue. In the Pittsburgh study (Casselbrant et al., 1999) lack of power did not allow the estimation of significant sex effects. The British study of same-sex twins did not report sex differences (Rovers et al., 2002). Due to lack of unlike-sex twins, neither of these studies were able to resolve the important question of sex differences in genetic influences. Thus, the recommendation of the authors of the former Norwegian study that their findings of sex differences in the heritability estimates needed replication from larger studies remains relevant (Kvaerner et al., 1997). 
The model fitting procedure in the present Norwegian study yielded almost equivalent fits for the two AE-models with and without common sexlimitation. According to the AIC criterion the common sex-limitation model yielded the best fit, suggesting that the magnitude of the genetic effect is larger in males. Despite differences among studies regarding the magnitude of genetic and environmental influences on otitis media our results lend greater support to findings suggesting sex differences in those influences. A plausible explanation of these sex differences may be that hormonal effects cause differences in the intrauterine environment, such that in utero levels of sex-steroids estradiol, progesterone and testosterone differ significantly (Sakai et al., 1991). A masculinising effect of androgens on the auditory system in females in unlike-sex twin pairs shown by McFadden (McFadden, 1993), suggest that hormonal influences cause different intrauterine environment for males and females.

\section{Limitations}

In the present study, selection bias cannot be completely ruled out. If, for instance, persons who are frequently ill tend to participate more often, recruitment bias may occur. However, selection bias for ear infections is not very likely in a large questionnaire on current health items that contain only a few questions on ear infections. Further, recall bias may occur in retrospective reports on childhood disease. Non-differential misclassification may also occur and artificially decrease all the correlation estimates. The loss of information and statistical power by the use of dichotomous and skewedly distributed variables has to be kept in mind in the interpretation of our findings. Estimated test-retest reliability and reporting consistency on the repeated questions on ear infections, suggesting sufficient validity of the measure, will be elucidated in a forthcoming publication (Kvestad et al., in prep).

The effect of co-twin contact and living together in their parental home on the correlation estimates have been reported for the subgroup of twins born in 1967-1974 in the former Norwegian study (Kvaerner et al., 1997). Co-twin contact did not significantly change the estimates, twin correlations were slightly higher in pairs who still lived at home and overall, little evidence of substantial bias effect was found. If any, a trend toward inflated correlations in both MZ and DZ twins may somewhat decrease the proportion between individual and common environment.

In conclusion, the present study adds new evidence of a substantial genetic effect from the same genetic source in males and females in addition to possible sex differences in heritability. Despite differences in data sets and methodological approaches between the British, American and Norwegian studies, there is agreement that the contribution of genetic factors to otitis media pathogenesis is substantial, confirming the need for studies of quantitative trait-loci. Twin studies may also play an important role in unravelling genetic mechanisms in otitis media, for instance by detecting genetic effects common to other diseases or characteristics, using multivariate designs.

\section{$\overline{\text { Acknowledgment }}$}

The Norwegian Institute of Public Health program of twin research is supported by grants from The Norwegian Research Council, The Norwegian Foundation for Health and Rehabilitation, the Nordic Council of Ministers Research Program in Longitudinal Epidemiology and the European Commission under the program "Quality of Life and Management of the Living Resources" of 5th Framework Program (no. QLG2-CT-2002-01254). We thank the twins for their participation.

\section{References}

Akaike, H. (1987). Factor-analysis and Aic. Psychometrika, 52, 317-332.

Casselbrant, M. L., Mandel, E. M., Fall, P. A., Rockette, H. E., Kurs-Lasky, M., Bluestone, C. D., et al. (1999). The heritability of otitis media: a twin and triplet study. Journal of the American Medical Association, 282, 2125-2130.

Daly, K. A., Casselbrant, M. L., Hoffman, H. J., Ingvarsson, L. B., Kvaerner, K. J., Tos, M., et al. (2002). Recent advances in otitis media. 2. Epidemiology, natural history, and risk factors. Annals of Otology, Rhinology and Laryngology (Supplement), 188, 19-25.

Harris, J. R., Magnus, P., \& Tambs, K. (2002). The Norwegian Institute of Public Health Twin Panel: a description of the sample and program of research. Twin Research, 5, 415-423.

Harris, J. R., Tambs, K., \& Magnus, P. (1995). Sexspecific effects for body mass index in the new Norwegian twin panel. Genetic Epidemiology, 12, 251-265.

Hartman, M., Rovers, M. M., Ingels, K., Zielhuis, G. A., Severens, J. L., \& van der Wilt, G. J. (2001). Economic evaluation of ventilation tubes in otitis media with effusion. Archives of Otolaryngology, Head and Neck Surgery, 127, 1471-1476.

Jaatinen, T., Ruuskanen, O., Truedsson, L., \& Lokki, M. L. (1999). Homozygous deletion of the CYP21ATNXA-RP2-C4B gene region conferring C4B deficiency associated with recurrent respiratory infections. Human Immunology, 60, 707-714.

Joki-Erkkila, V. P., Puhakka, H., \& Hurme, M. (2002). Cytokine gene polymorphism in recurrent acute otitis media. Archives of Otolaryngology, Head and Neck Surgery, 128, 17-20.

Jöreskog K.G., \& Sörbom D. (2000). Prelis 2 Users reference guide [Computer software]. Scientific Software International, Inc. 
Kvaerner, K. J., Nafstad, P., \& Jaakkola, J. J. (2002). Otolaryngological surgery and upper respiratory tract infections in children: an epidemiological study. Annals of Otology, Rhinology and Laryngology, 111, 1034-1039.

Kvaerner, K. J., Tambs, K., Harris, J. R., \& Magnus, P. (1997). Distribution and heritability of recurrent ear infections. Annals of Otology, Rhinology and Laryngology, 106, 624-632.

Kvestad E., Kvaerner, K. J., Røysamb E., Tambs, K., Harris, J. R., \& Magnus P. (In preparation). Heritability of otitis media: Reliability and validity of retrospective twin data.

Lahti, M., Lofgren, J., Marttila, R., Renko, M., Klaavuniemi, T., Haataja, R., et al. (2002). Surfactant protein $\mathrm{D}$ gene polymorphism associated with severe respiratory syncytial virus infection. Pediatric Research, 51, 696-699.

Lofgren, J., Ramet, M., Renko, M., Marttila, R., \& Hallman, M. (2002). Association between surfactant protein A gene locus and severe respiratory syncytial virus infection in infants. Journal of Infectious Diseases, 185, 283-289.
McFadden, D. (1993). A masculinizing effect on the auditory systems of human females having male co-twins. Proceedings of the National Academy of Sciences of the United States of America, 90, 11900-11904.

Neale, M. C., Boker, S. M., Xie, G., \& Maes, H. H. (1999). Mx: statistical Modeling. (5th ed.) VCU Box 9000126, Richmond, VA 23298: Department of Psychiatry.

Neale, M. C. \& Cardon, L. R. (1992). Methodology for genetic studies of twins and families. (1st ed.) Boston.

Niemela, M., Uhari, M., Mottonen, M., \& Pokka, T. (1999). Costs arising from otitis media. Acta Paediatrica, 88, 553-556.

Rovers, M., Haggard, M., Gannon, M., KoeppenSchomerus, G., \& Plomin, R. (2002). Heritability of symptom domains in otitis media: a longitudinal study of 1,373 twin pairs. American Journal of Epidemiology, 155, 958-964.

Sakai, L. M., Baker, L. A., Jacklin, C. N., \& Shulman, I. (1991). Sex steroids at birth: genetic and environmental variation and covariation. Developmental Psychobiology, 24, 559-570. 